\title{
Generating Function Associated with the Rational Solutions of the Painlevé II Equation
}

\author{
Katsunori Iwasaki, Kenji Kajiwara and Toshiya Nakamura \\ Graduate School of Mathematics \\ Kyushu University \\ 6-10-1 Hakozaki, Higashi-ku, Fukuoka 812-8581 Japan
}

\begin{abstract}
We consider the Hankel determinant representation for the rational solutions of the Painlevé II equation. We give an explicit formula for the generating function of the entries in terms of logarithmic derivative of the Airy function, which by itself is a particular solution of the Painlevé II equation.
\end{abstract}

Key words: Painlevé II equationrational solutions Hankel determinant, generating functionAiry function.

2000 Mathematics Subject Classification: 33C10, 33E17, 34M55

\section{Introduction}

In this paper we introduce a generating function associated with the rational solutions of the Painlevé II equation and characterize it explicitly in terms of the Airy function. Here the Painlevé II equation $\left(\mathrm{P}_{\mathrm{II}}\right)$ is a second order nonlinear ordinary differential equation with a parameter $\alpha$,

$$
\frac{d^{2} v}{d x^{2}}=2 v^{3}-4 x v+4 \alpha
$$

This equation has exactly one rational solution for $\alpha$ being an arbitrary integer [1] and has no rational solution if $\alpha$ is not an integer [5, 6, 10]. It admits a Bäcklund transformation $(v, \alpha) \mapsto(-v,-\alpha)$, which clearly maps rational solutions to rational solutions. Moreover the unique rational solution 
for $\alpha=0$ is the trivial solution $v \equiv 0$. Hence $\alpha$ may be restricted to positive integers without loss of generality.

According to [2], any rational solution admits two types of determinant representation; one is the Jacobi-Trudi type and the other is the Hankel type, the latter being described as follows: For each positive integer $N+1$, the unique rational solution for $\alpha=N+1$ is given by

$$
v=\frac{d}{d x} \log \frac{\sigma_{N+1}}{\sigma_{N}}
$$

where $\sigma_{N}$ is the Hankel determinant

$$
\sigma_{N}=\left|\begin{array}{cccc}
a_{0} & a_{1} & \cdots & a_{N-1} \\
a_{1} & a_{2} & \cdots & a_{N} \\
\vdots & \vdots & \ddots & \vdots \\
a_{N-1} & a_{N} & \cdots & a_{2 N-2}
\end{array}\right|
$$

with $a_{n}=a_{n}(x)$ being polynomials defined by the recurrence relation

$$
\begin{aligned}
a_{0} & =x, \quad a_{1}=1, \\
a_{n+1} & =\frac{d a_{n}}{d x}+\sum_{k=0}^{n-1} a_{k} a_{n-1-k} .
\end{aligned}
$$

On one hand, the determinant representation of the Jacobi-Trudi type implies that the rational solutions can be described in terms of certain specializations of Schur polynomials [2]. What, on the other hand, does the determinant representation of the Hankel type mean? Or more simply we may ask: What is the sequence $a_{n}$ ?

As will be seen in this paper, an answer to the above question is very intriguing! The generating function for the sequence $a_{n}$ is essentially the Airy function. More precisely we have the following:

Theorem 1.1 Let $\theta(x, t)$ be an entire function of two variables defined by

$$
\theta(x, t)=\exp \left(2 t^{3} / 3\right) \operatorname{Ai}\left(t^{2}-x\right)
$$

where $\operatorname{Ai}(z)$ is the Airy function. Then there exists an asymptotic expansion

$$
\frac{\partial}{\partial t} \log \theta(x, t) \sim \sum_{n=0}^{\infty} a_{n}(x)(-2 t)^{-n}
$$

as $t \rightarrow \infty$ in any proper subsector of the sector $|\arg t|<\pi / 2$. 
The occurrence of the Airy function in Theorem 1.1 is quite suggestive to those who know that $\mathrm{P}_{\mathrm{II}}$ (1) has a particular solution

$$
v=\frac{d}{d x} \log \operatorname{Ai}\left(2^{1 / 3} x\right), \quad \alpha=1 / 2 .
$$

As is well known, $\mathrm{P}_{\mathrm{II}}$ (11) admits (exactly) two classes of classical solutions, namely, the class of rational solutions and that of Airy function solutions [10]. Theorem 1.1 might then be interpreted as asserting that all members of the former class are generated by a particular member of the latter; see $\S$ 田 for further discussions.

\section{Riccati Equation}

We will find out a differential equation satisfied by the generating function

$$
F(x, t)=\sum_{n=0}^{\infty} a_{n}(x)(-2 t)^{-n}
$$

which is thought of as a formal power series of $t^{-1}$ with polynomial coefficients in $x$. To this end we have to deduce some other recurrence relations for $a_{n}$ from the original one (2) together with the data on the first three terms

$$
a_{0}=x, \quad a_{1}=1, \quad a_{2}=x^{2} .
$$

Lemma 2.1 The infinite sequence $a_{n}$ satisfies recurrence relations

$$
\begin{aligned}
\frac{d a_{n+1}}{d x} & =2 n a_{n-1} \\
a_{n+1} & =2(n-1) a_{n-2}+\sum_{k=0}^{n-1} a_{k} a_{n-k-1} .
\end{aligned}
$$

Proof. Recurrence relation (7) was already mentioned in [2], (58)], but a proof is included here for the sake of completeness. The proof proceeds by induction on $n$. Assume that (7) holds for $1, \ldots, n$. Recurrence relation (2) and induction hypothesis lead to

$$
a_{n+2}=a_{n+1}^{\prime}+\sum_{k=0}^{n} a_{k} a_{n-k}=2 n a_{n-1}+\sum_{k=0}^{n} a_{k} a_{n-k}
$$


Differentiating both sides and using the initial condition (6), induction hypothesis and the recurrence relation (2), one finds

$$
\begin{aligned}
a_{n+2}^{\prime}= & 2 n a_{n-1}^{\prime}+2 a_{n}+\sum_{k=2}^{n} a_{k}^{\prime} a_{n-k}+\sum_{k=0}^{n-2} a_{k} a_{n-k}^{\prime} \\
= & 2 n a_{n-1}^{\prime}+2 a_{n}+\sum_{k=2}^{n} 2(k-1) a_{k-2} a_{n-k} \\
& +\sum_{k=0}^{n-2} a_{k} \cdot 2(n-k-1) a_{n-k-2} \\
= & 2 a_{n}+2 n a_{n-1}^{\prime}+\sum_{k=0}^{n-2} 2 n a_{k} a_{n-k-2} \\
= & 2 a_{n}+2 n a_{n} \\
= & 2(n+1) a_{n} .
\end{aligned}
$$

Hence (7) holds for $n+1$ and the induction is complete. Recurrence relation (8) is obtained by substituting (7) into (2).

Lemma 2.1 leads to a differential equation of the Riccati type.

Lemma 2.2 The generating function $F(x, t)$ satisfies a differential equation

$$
t \frac{\partial f}{\partial t}+t f^{2}-\left(4 t^{3}+1\right) f+4 x t^{3}-2 t^{2}=0 .
$$

Proof. From (5) one has

$$
\begin{aligned}
t F^{2} & =t \sum_{n=0}^{\infty}\left(\sum_{k=0}^{n} a_{k} a_{n-k}\right)(-2 t)^{-n} \\
& =t x^{2}+t \sum_{n=1}^{\infty}\left(\sum_{k=0}^{n} a_{k} a_{n-k}\right)(-2 t)^{-n} \\
& =t x^{2}+t \sum_{n=1}^{\infty}\left(a_{n+2}-2 n a_{n-1}\right)(-2 t)^{-n} \\
& =t x^{2}+4 t^{3} \sum_{n=3}^{\infty} a_{n}(-2 t)^{-n}+\sum_{n=0}^{\infty}(n+1) a_{n}(-2 t)^{-n} \\
& =t x^{2}+4 t^{3}\left\{F-x-(-2 t)^{-1}-x^{2}(-2 t)^{-2}\right\}+\left(-t \frac{\partial}{\partial t}+1\right) F \\
& =-t \frac{\partial F}{\partial t}+\left(4 t^{3}+1\right) F-4 x t^{3}+2 t^{2}
\end{aligned}
$$


where (6) was used in the second and fifth equalities and (8) in the third equality. Hence $F$ satisfies the differential equation (9).

\section{The Airy Function}

The Riccati equation (9) can be linearized in a standard manner.

Lemma 3.1 The change of dependent variable

$$
f=\frac{\partial}{\partial t} \log \left\{u \exp \left(2 t^{3} / 3\right)\right\}
$$

transforms the Riccati equation (9) into a linear equation

$$
t \frac{\partial^{2} u}{\partial t^{2}}-\frac{\partial u}{\partial t}-4\left(t^{5}-x t^{3}\right) u=0
$$

Furthermore the change of independent variable

$$
z=t^{2}-x
$$

simplifies (11) into the Airy differential equation

$$
\frac{\partial^{2} u}{\partial z^{2}}-z u=0
$$

The proof is just by direct calculations.

It is well known 4 , 11] that the Airy equation (13) has the formal solutions

$$
U_{ \pm}(z)=\frac{1}{2 \sqrt{\pi}} \exp \left( \pm \frac{2 z^{3 / 2}}{3}\right) z^{-1 / 4} \sum_{n=0}^{\infty} \frac{(1 / 6)_{n}(5 / 6)_{n}}{n !}\left( \pm \frac{4 z^{3 / 2}}{3}\right)^{-n}
$$

and that the Airy function $\operatorname{Ai}(z)$ admits an asymptotic representation

$$
\operatorname{Ai}(z) \sim U_{-}(z)
$$

as $z \rightarrow \infty$ in any proper subsector of the sector $|\arg z|<\pi$.

Lemma 3.2 Equation (11) admits a formal solution

$$
U(x, t)=\frac{1}{2 \sqrt{\pi}} \exp \left(-\frac{2 t^{3}}{3}\right) t^{-1 / 2} \exp \left\{\frac{1}{2} \sum_{n=1}^{\infty} \frac{a_{n+1}(x)}{n}(-2 t)^{-n}\right\}
$$

If the branch $z^{1 / 2}=t\left(1-x t^{-2}\right)^{1 / 2}=t-(x / 2) t^{-1}+\cdots$ is taken for the square root of (12), then

$$
U(x, t)=U_{-}(z)
$$


Proof. Observe that $u=U(x, t)$ defined by (16) satisfies the equation (10) with $f=F(x, t)$. Since $F(x, t)$ solves the Riccati equation (9), Lemma 3.1 implies that (16) solves the linear equation (11). Under the relation (12), $U(x, t)$ must be either $U_{+}(z)$ or $U_{-}(z)$ since it is a formal solution of the Airy equation (13). Comparing the exponential factors of (14) and (16), we must choose the minus sign and hence have (17).

Proof of Theorem 1.1. We are now in a position to establish Theorem 1.1. By (15) and (17) the function $\mathrm{Ai}\left(t^{2}-x\right)$ has an asymptotic representation $U(x, t)$ as $t \rightarrow \infty$ in any proper subsector of the sector $|\arg t|<\pi / 2$ (note that under the relation (12) a sector of central angle $\theta$ in the $z$ plane corresponds to one of central angle $\theta / 2$ in the $t$ plane). This fact, through the transformation (10), leads to the asymptotic expansion (田).

\section{Concluding Discussions}

In this paper we have considered the infinite sequence of polynomials that appears in the Hankel determinant representation for rational solutions of the Painleve II equation. As a result we have constructed a generating function explicitly represented in terms of the Airy function, which by itself is a particular solution of the Painleve II equation.

It is natural to ask whether there are similar phenomena for other Painlevé equations. It is known that any other Painlevé equation, except for the first one $\mathrm{P}_{\mathrm{I}}$, also admits two classes of classical solutions, namely, the class of rational solutions and that of special function solutions; Bessel for $\mathrm{P}_{\mathrm{III}}$, Hermite-Weber for $\mathrm{P}_{\mathrm{IV}}$, Kummer for $\mathrm{P}_{\mathrm{V}}$ and Gauss for $\mathrm{P}_{\mathrm{VI}}$ [6, 7, 8, 9]. It is also known that all the rational solutions are represented in terms of Hankel determinants with entries from an infinite sequence of certain special polynomials [3]. Now a natural question is whether this infinite sequence has a generating function expressible in a closed form by using the respective special functions. Moreover, how about generic (transcendental) solutions? Do they admit similar phenomena? Further, how about the discrete cases? These points, together with the mechanism behind these strange phenomena, are yet to be explored in the future.

\section{References}

[1] H. Airault, Rational solutions of Painlevé equations, Stud. Appl. Math. 61 (1) (1979), 31-53. 
[2] K. Kajiwara and Y. Ohta, Determinant structure of the rational solutions for the Painlevé II equation, J. Math. Phys. 37 (9) (1996), 46934704 .

[3] K. Kajiwara, T. Masuda, M. Noumi, Y. Ohta and Y. Yamada, Determinant formula for the Toda and discrete Toda equations, Funkcial. Ekvac. 44 (2) (2001), 291-308.

[4] N. N. Lebedev, Special functions and their applications, Prentice-Hall, Englewood Cliffs, 1965.

[5] Y. Murata, Rational solutions of the second and the fourth Painlevé equations, Funkcial. Ekvac. 28 (1) (1985), 1-32.

[6] K. Okamoto, Studies on the Painlevé equations I, Sixth Painlevé equations $P_{\mathrm{VI}}$, Ann. Mat. Pura Appl. 146 (4) (1987), 337-381.

[7] K. Okamoto, Studies on the Painlevé equations II, Fifth Painlevé equations $P_{\mathrm{V}}$, Japan. J. Math. (N.S.) 13 (1) (1987), 47-76.

[8] K. Okamoto, Studies on the Painlevé equations III, Second and fourth Painlevé equations, $P_{\mathrm{II}}$ and $P_{\mathrm{IV}}$, Math. Ann. 275 (1986), 222-254.

[9] K. Okamoto, Studies on the Painlevé equations IV, Third Painlevé equations $P_{\mathrm{III}}$, Funkcial. Ekvac. 30 (2-3) (1987), 305-332.

[10] H. Umemura and H. Watanabe, Solutions of the second and fourth Painlevé equations I, Nagoya Math. J. 148 (1997), 151-198.

[11] W. Wasow, Asymptotic expansions for ordinary differential equations, Interscience Publ., New York, 1965. 